\title{
The QCD vacuum as a disordered medium: A simplified model for the QCD Dirac operator
}

\author{
Antonio M. García-García \\ Laboratoire de Physique Théorique et Modèles Statistiques, Bât. 100, \\ Université de Paris-Sud, 91405 Orsay Cedex, France \\ James C. Osborn \\ Physics Department, University of Utah, Salt Lake City, UT 84112, USA
}

\begin{abstract}
We model the QCD Dirac operator as a power-law random banded matrix (RBM) with the appropriate chiral symmetry. Our motivation is the form of the Dirac operator in a basis of instantonic zero modes with a corresponding gauge background of instantons. We compare the spectral correlations of this model to those of an instanton liquid model (ILM) and find agreement well beyond the Thouless energy. In the bulk of the spectrum the (dimensionless) Thouless energy of the RBM scales with the square root of system size in agreement with the ILM and chiral perturbation theory. Near the origin the scaling of the (dimensionless) Thouless energy in the RBM remains the same as in the bulk which agrees with chiral perturbation theory but not with the ILM. Finally we discuss how this RBM should be modified in order to describe the spectral correlations of the QCD Dirac operator at the finite temperature chiral restoration transition.

PACS numbers: 72.15.Rn, 71.30.+h, 05.45.Df, 05.40.-a
\end{abstract}

In recent years the relation between spontaneous chiral symmetry breaking $(\mathrm{S} \chi \mathrm{SB})$ in $\mathrm{QCD}$ and the phenomenon of conductivity in a disordered medium has been investigated in the literature [1, 2, 3]. In the latter, conductivity is produced by electrons that although initially bound to impurities may get delocalized by orbital overlapping. In QCD the quark zero modes initially bound to an instanton get delocalized due to the strong overlap with other would-be zero modes and consequently chiral symmetry is broken. In the case of atoms the overlap is effective only among nearest neighbors due to the exponential decay of the electron wavefunction. However, in the QCD vacuum, the decay is power-law and long range hopping is possible. Thus even if one assumes a perfect trade between impurities and instantons and between electrons and quarks, the associated Anderson model should posses long range hopping. Such models have already been discussed in the literature [4, 5]. The main conclusion of these works was that power-law hopping may induce a metal insulator transition even in one dimensional systems if the exponent of the hopping decay matched the dimension of the space. Similar findings were also reported in a related model, a random banded matrix with a power-law decay [6, 7].

Random matrix techniques have already been used in the context of QCD. In the infrared limit the eigenvalue correlations of the QCD Dirac operator do not depend on the dynamical details of the QCD Lagrangian but only on the global symmetries of the QCD partition function [8]. Thus random matrices with the correct chiral symmetry of QCD (termed chiral random matrices) [9] accurately describe the spectral properties of the QCD Dirac operator up to some scale known as the Thouless energy. For larger energy differences dynamic features become im- portant and the standard random matrix model ceases to be applicable.

In this letter we study an improved matrix model for QCD which reproduces features of the eigenvalue spectrum beyond the Thouless energy. We want to incorporate the phenomenological fact that the matrix elements of the QCD Dirac operator in a basis of zero modes decay as a power of the instanton-anti-instanton distance into the random matrix approximation. We thus propose a chiral RBM as an effective model of the QCD Dirac operator. We first give a brief introduction on the role of instantons in the QCD vacuum. Next we introduce the chiral RBM and describe its main properties at both the origin and bulk of the spectrum. In the latter case an analytical treatment is possible. Then we compare the spectral correlations of this model with the results of an instanton liquid simulation. Finally we discuss how the RBM can be modified to describe the spectral correlations of the QCD Dirac operator at the finite temperature chiral restoration transition.

\section{S $\chi$ SB FROM INSTANTONS}

The discovery of instantons has had a large impact on our understanding of non-perturbative aspects of QCD [10] (for a modern review see 11]). Instantons are classical solutions of the Yang-Mills equations of motion in Euclidean space. An important property is that the Euclidean QCD Dirac operator has an exact zero eigenvalue in the field of an instanton. The spectral properties of the low lying modes of the Dirac operator are thus controlled by these non-perturbative configurations.

Unfortunately the construction of a consistent QCD 
vacuum based on instantons faces serious technical difficulties. Exact analytical multi-instanton configurations are hard to obtain since the Yang-Mills equations of motion for QCD are nonlinear and therefore a superposition of single instanton contributions is not itself a solution. Additionally quantum corrections may spoil the semiclassical picture implicitly assumed of a QCD vacuum composed of instantons well separated and weakly interacting. These problems have been overcome either by invoking variational principles [12] or by phenomenologically fixing certain parameters of the instanton ensemble. The latter case, usually referred to as the instanton liquid model (ILM) 13], yields accurate estimates of vacuum condensates and hadronic correlation functions 14 just by setting the density of instantons to be $N / V \approx 1 \mathrm{fm}^{-4}$ and the mean size $\bar{\rho} \approx 1 / 3 \mathrm{fm}$. Lattice simulations have also supported the picture of a $\mathrm{QCD}$ vacuum dominated by instantons [15].

An advantage of these models is that they satisfactorily explain how chiral symmetry is spontaneously broken in the QCD vacuum [12. The chiral condensate (the order parameter signaling $\mathrm{S} \chi \mathrm{SB})\langle\bar{\psi} \psi\rangle$ is related to the spectral density $\rho(\epsilon)$ of the QCD Dirac operator around zero through the Banks-Casher relation [16],

$$
\langle\bar{\psi} \psi\rangle=-\lim _{\epsilon \rightarrow 0} \lim _{V \rightarrow \infty} \frac{\pi \rho(\epsilon)}{V},
$$

where $V$ is the space-time volume. The ILM provides a phenomenological model for these low energy modes of the QCD Dirac operator. In a basis of $N / 2$ instantonic zero modes $\psi_{I}\left(\vec{z}_{i}\right)$ and $N / 2$ anti-instantonic zero modes $\psi_{A}\left(\vec{z}_{j}\right)$ the matrix elements of the Dirac operator take the form (for zero quark mass)

$$
\mathcal{D}_{I L M}=\left(\begin{array}{cc}
0 & i T_{I A} \\
i T_{I A}^{\dagger} & 0
\end{array}\right)
$$

with the $N / 2 \times N / 2$ overlap matrix given by

$$
T_{I A}=\left\langle\psi_{I}\left(\vec{z}_{i}\right)|\not D| \psi_{A}\left(\vec{z}_{j}\right)\right\rangle \sim \rho_{i} \rho_{j} /\left|\vec{R}_{i j}\right|^{3}
$$

for large separations $\vec{R}_{i j}=\vec{z}_{i}-\vec{z}_{j}$ between the center of instanton $i$ and anti-instanton $j$ with $\rho_{i}$ and $\rho_{j}$ their sizes. Due to the chirality of the zero modes only matrix elements connecting an instanton with an anti-instanton do not vanish. Physically the amplitude of the matrix elements $T_{I A}$ represents the probability for a quark to hop between an instanton and an anti-instanton. An isolated (anti-)instanton would cause an exact zero mode but this degeneracy is lifted through overlap with neighboring instantons. Thus the would-be zero modes are effectively split around zero. As more (anti-)instantons are added a continuous band spectrum is formed with a spectral density finite at zero. As mentioned previously, it is precisely this dynamically generated non-zero spectral density that causes, through the Bank-Casher relation, a finite value of the chiral condensate.

\section{THE CHIRAL RANDOM BANDED MODEL}

Here we study the spectral properties of an ensemble of chiral random Hermitian $N \times N$ matrices given by

$$
\mathcal{D}_{R B M}=\left(\begin{array}{cc}
0 & C \\
C^{\dagger} & 0
\end{array}\right)
$$

where $C$ is a $N / 2 \times N / 2$ complex matrix with independently distributed Gaussian variables with zero mean. The variance of the matrix elements $C_{i j}$ are chosen to decay as a power of $r=|i-j|$ which measures the distance from the diagonal. Since the ILM uses periodic boundary conditions we use a periodic form of the power-law decay [17] given by

$$
\left\langle\left|C_{i j}\right|^{2}\right\rangle=\left\{1+\left[\frac{\sin (2 \pi r / N)}{\pi b / N}\right]^{2 \alpha}\right\}^{-1}
$$

where $\alpha$ and $b$ are real parameters. The choice of complex matrix elements corresponds to a matrix model with a unitary symmetry which is appropriate for QCD with the phenomenologically relevant $S U(3)$ color group. Due to the chiral symmetry, the eigenvalues of (4) come in pairs of $\pm \epsilon_{i}$. This feature induces an additional level repulsion around zero which results in different spectral correlations for eigenvalues near zero (the origin) and away from zero (the bulk).

In the bulk the spectral correlations should not be affected by the block structure and should coincide with the non-chiral version of (44) which has been intensively studied in recent years [7, 17. The use of the supersymmetry method [18] permits an analytical evaluation of both spectral properties and eigenfunction statistics [7] in a certain region of parameters. In the thermodynamic limit the eigenfunctions are multifractal for $\alpha=1$ and localized (delocalized) for $\alpha>1(\alpha<1)$ respectively [7]. The spectral correlations in the $g=E_{c} / \Delta>>1\left(E_{c}\right.$ is the Thouless energy and $\Delta$ is the mean level spacing) limit can be expressed through the spectral determinant of a classical diffusion operator [19]. The two point correlation function is defined as $R_{2}(s)=\Delta^{2}\langle\rho(\epsilon) \rho(\epsilon+s \Delta)\rangle-1$ where $\rho(\epsilon)$ is the density of states at energy $\epsilon$ and the average is over an ensemble of RBM. For the unitary ensemble (our case)

$$
R_{2}(s)=-\frac{1}{4 \pi^{2}} \frac{\partial^{2}}{\partial s^{2}} \ln \frac{D(s, g)}{s^{2}}+\frac{\cos (2 \pi s)}{2 \pi^{2} s^{2}} D(s, g) .
$$

Due to the power-law decay, the spectral determinant $D(s, g)=\prod_{n \neq 0}\left(1+s^{2} / \epsilon_{n}^{2}\right)^{-1}\left(\epsilon_{n}=g|n|^{2 \alpha-1}\right)$ corresponds with a process of anomalous diffusion [7]. For $1 / 2<\alpha<1$ the dimensionless conductance increases with the system size as $g=C_{\alpha}(b) N^{2-2 \alpha}$ with $C_{\alpha}(b)$ a known constant. The scaling of $g$ thus resembles that of a weakly disordered conductor in $d=2 /(2 \alpha-1)$ dimensions [7]. 


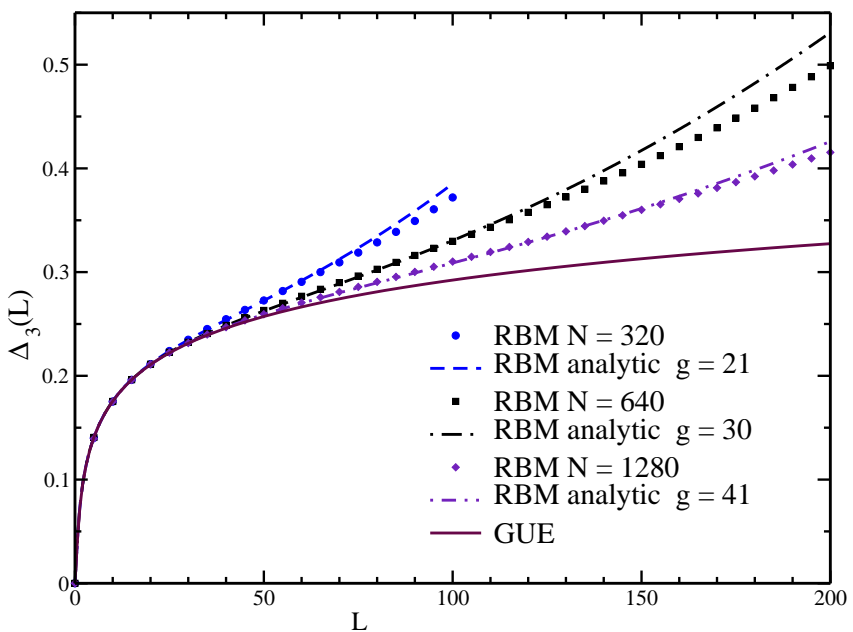

FIG. 1: Spectral rigidity for the RBM in the bulk. Points correspond to the numerical simulations of the chiral RBM for $b=1$. Lines correspond to the analytical result of the non-chiral RBM (6) for the given values of $g$.

The above results were derived for the non-chiral RBM. As mentioned above they are also expected to describe the chiral RBM (4) in the bulk region. We have chosen the scale of $b$ in (4) to agree with results for the nonchiral model. Strictly speaking, the factor $C_{\alpha}(b)$ was derived [] for $b \gg 1$ and it is not clear how the result should be modified for smaller $b$. Below we will show that in fact the analytical results agree very well even at $b=1$. Unlike in the bulk, analytical results for the spectral correlations close to the origin are not known. They can be obtained by modifying the supersymmetry method to account for the chiral structure. This issue will be postponed to a future publication [20] and in this letter we will rely on numerical calculations for the study of the spectral correlations close to the origin. Lastly we remark that though the power-law decay in the instanton liquid is not in principle related to the matrix index, as in our matrix model, numerical simulations show that both models yield similar results [6]. We stick to (4) due to the availability of analytical results.

Given the known results for the non-chiral RBM we can now choose the parameter $\alpha$. Recall that our motivation to propose a random banded model is the decay of the ILM overlap matrix elements (3). It was shown in [5] that the spectral properties of systems with power-law hopping are similar in different dimensions provided that the decay exponent equaled the dimension. Since in the ILM the decay exponent (three) is less than the dimension (four) we expect this to map onto a 1D RBM model with $\alpha<1$. We choose $\alpha=3 / 4$ because the volume dependence of the dimensionless conductance (also called dimensionless Thouless energy) in this case $g \approx 1.17 \sqrt{b N}$ 7] coincides with what is expected for QCD according to chiral perturbation theory 21. As mentioned above, the spectral properties of the RBM at $\alpha=3 / 4$ are similar to those of a disordered conductor in four dimensions.

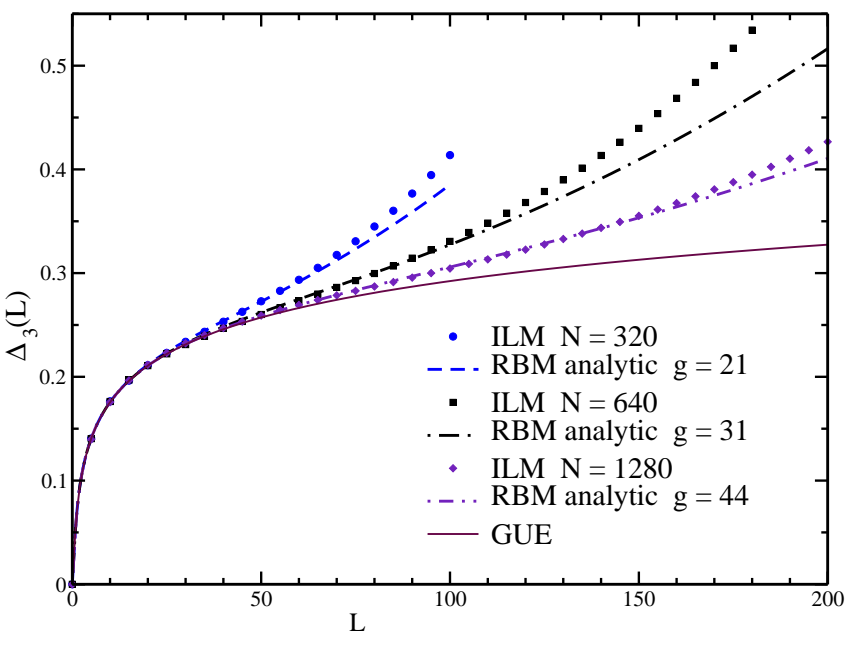

FIG. 2: Spectral rigidity for the ILM and RBM in the bulk. Points correspond to numerical simulation of the ILM. Lines correspond to the analytical result of the non-chiral RBM (6) for the given values of $g$.

\section{RESULTS}

We generated sets of matrices for the RBM and the ILM and calculated their eigenvalues in order to compare the spectral correlations. The results of each simulation are averaged over $10^{4}$ configurations. For both models the simulations were done in the quenched approximation which allowed us to use values of $N$ ranging from 320 up to 1280 . For the ILM we used the same model studied in [1] including the standard density of $N / V=1 \mathrm{fm}^{-4}$. Additional details can be found in [11].

We first check that the result (6), derived for the nonchiral RBM, agrees with the chiral result in the bulk. The analytical formula is actually divergent for $\alpha=3 / 4$ and needs a cutoff in the spectral determinant $D(s, g)$. If we calculate the number variance which is given by $\Sigma^{2}(L)=L+2 \int_{0}^{L}(L-s) R_{2}(s) d s$ we find that it is strongly cutoff dependent. Instead we look at the spectral rigidity $\Delta_{3}(L)=\frac{2}{L^{4}} \int_{0}^{L}\left(L^{3}-2 L^{2} x+x^{3}\right) \Sigma^{2}(x) d x$ which is not sensitive to the cutoff.

In Figure 1 we show the spectral rigidity in the bulk of the chiral RBM obtained from numerical simulation at $b=1$ along with the analytical formula of the nonchiral model for different values of $g$. The values of $g$ were chosen by eye to match the numerical results for the corresponding $N$. We find good agreement with the theoretical expectation $g \approx 1.17 \sqrt{N}$ within about $2 \%$.

In Figure 2 we compare the spectral rigidity in the bulk of the ILM with analytical predictions of the RBM. Again the values of $g$ for each volume were chosen by eye to closely match the ILM. The analytical results using these values of $g$ agree well with the ILM up to a scale of about $30 \%$ of the number of positive eigenvalues $(N / 2)$. The values of $g$ scale closely to $g \approx 1.23 \sqrt{N}$ except for the smallest volume $(N=320)$ where a $5 \%$ deviation is observed. This corresponds to $b \approx 1.1$. The observed scaling of the dimensionless Thouless energy in the chiral 


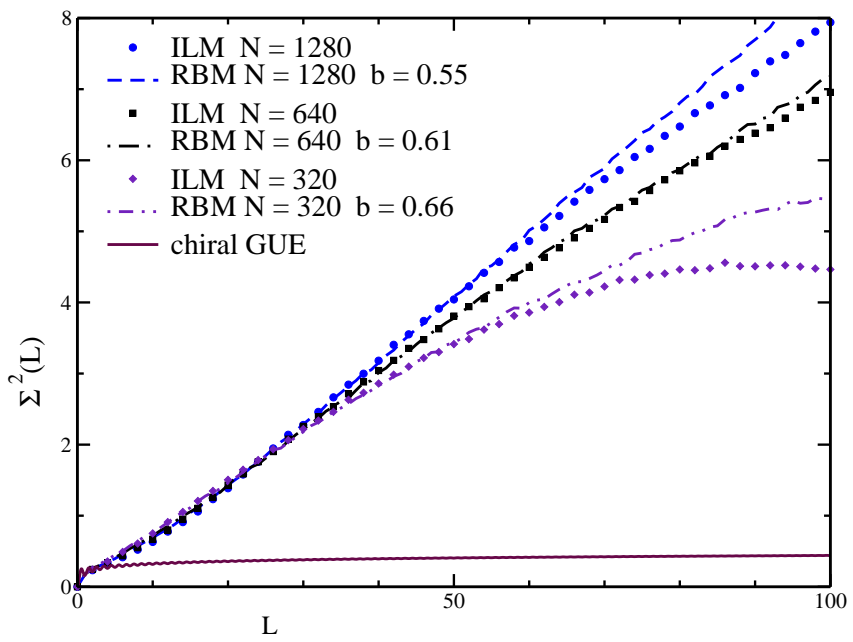

FIG. 3: Number variance $\Sigma^{2}(L)$ close to the origin. Points correspond to numerical simulations of the ILM. Lines correspond to numerical simulations of the chiral RBM for the given values of $b$.

$\mathrm{RBM}$ is in agreement with theoretical predictions [1, 21. and lattice results 22] for QCD.

We do not yet have analytical results for the spectral correlations of the RBM at the origin and therefore rely solely on numerical simulations. In Figure 3 we show the number variance near the origin for the ILM and chiral RBM. The corresponding values of $b$ for the RBM were also chosen by eye to provide a good fit. The agreement between both models is very good up to about 50 eigenvalues but we could not fit all volumes with a single $b$. The reason is that the chiral RBM has the same $g \sim \sqrt{N}$ scaling as in the bulk while the instanton liquid shows a weaker volume dependence. We find the scaling $b \approx 1.4 N^{-0.13}$ which gives $g \approx 1.4 N^{0.43}$ for the ILM. It would be interesting to compare these results with lattice simulations where a $g \sim \sqrt{N}$ scaling has also been reported close to the origin.

Finally we mention how the chiral RBM should be modified to describe QCD at finite temperature. As usual in field theory, temperature is introduced by compactifying one of the spatial dimensions. Thus the effect of temperature in Euclidean QCD is to reduce the effective dimensionality of the system to three. Now since the effective dimension of the space matches the power-law decay of the QCD Dirac operator $\left(\sim 1 / R^{3}\right)$ one expects, according to [5, 6], multifractal wavefunctions typical of a metal-insulator transition. The same chiral RBM proposed in this paper may be used in this situation but with $\alpha=1$ (see [23] for a model with similar spectral properties). The above arguments suggest that if the restoration of chiral symmetry at finite temperature is dominated by instantons, the physical mechanism leading to the quark-gluon plasma state of matter would be similar to a metal-insulator transition. Clearly further work is needed to explore this exciting relation.

To conclude, we have proposed a chiral random banded model with power-law decay in order to describe the spec- tral correlations of the QCD Dirac operator beyond the Thouless energy. We have thus combined the asymptotic power-law tail observed in instanton liquid models with the random matrix approach valid for small spacings. We have provided numerical evidence that the resulting chiral RBM does (at least for the two-point function) describe the spectral correlations of the QCD Dirac operator well beyond the Thouless energy. Finally we have mentioned that at the finite temperature chiral restoration transition the appropriately modified chiral RBM predicts a metal-insulator behavior including multifractal wavefunctions and the physics of the Anderson transition in the QCD vacuum.

We thank J.J.M Verbaarschot for illuminating discussions. A.M.G. was supported by the EU network "Mathematical aspects of quantum chaos". J.C.O. was supported in part by NSF PHY 01-39929.

[1] J.C. Osborn and J.J.M. Verbaarschot, Phys. Rev. Lett. 81268 (1998); Nucl. Phys. B525 738 (1998).

[2] R.A. Janik, M.A. Nowak, G. Papp, and I. Zahed, Phys. Rev. Lett. 81264 (1998).

[3] D. Diakonov and P. Petrov, Nucl. Phys. B272 457 (1986); Phys. Lett. B147 351 (1984); hep-ph/9602375.

[4] G. Yeung and Y. Oono, Europhys. Lett. 41061 (1987).

[5] L.S. Levitov, Phys. Rev. Lett. 64547 (1990).

[6] A. Parshin and H.R. Schober, Phys. Rev. B 5710232 (1998).

[7] A.D. Mirlin, et. al., Phys. Rev. E 543221 (1996).

[8] E. Shuryak and J.J.M. Verbaarschot, Nucl. Phys. A560 306 (1993).

[9] J.J.M Verbaarschot, Phys. Rev. Lett. 722531 (1994).

[10] A. Belavin, A. Polyakov, A. Schwartz, and Y. Tyupkin, Phys. Lett. 5985 (1975); G. 't Hooft, Phys. Rev. Lett. 378 (1976).

[11] T. Schäfer and E. Shuryak, Rev. Mod. Phys. 70323 (1998).

[12] D. Diakonov and V. Petrov, Nucl. Phys. B245 259 (1984).

[13] E. Shuryak, Nucl. Phys. B203 93,116,140 (1982).

[14] T. Shafer, E. Shuryak and J.J.M. Verbaarschot, Nucl. Phys. B412 143 (1994).

[15] M.-C. Chu, et. al., Phys. Rev. D 496039 (1994); C. Michael and P.S. Spencer, Phys. Rev. D 524691 (1995).

[16] T. Banks and A. Casher, Nucl. Phys. B169 103 (1980).

[17] F. Evers and A.D. Mirlin, Phys. Rev. Lett. 843690 (2000); E. Cuevas, et.al., Phys. Rev. Lett. 88016401 (2002); I. Varga and D. Braum, Phys. Rev. B 61 R11859 (2000).

[18] K.B. Efetov, Adv. Phys. 3253 (1983).

[19] A. V. Andreev and B. L. Altshuler, Phys. Rev. Lett. 75 902 (1995).

[20] A.M. Garcia-Garcia and K. Takahashi, In preparation.

[21] J. Gasser and H. Leutwyler, Phys. Lett. 188B 477 (1987); Nucl. Phys. B307 763 (1988).

[22] M.E. Berbenni-Bitsch, et. al., Phys. Lett. B 43814 (1998); M. Gockeler, et. al., Phys. Rev. D59 094503 (1999);

[23] A.M. Garcia-Garcia and J.J.M. Verbaarschot, Nucl. Phys. B586 668 (2000). 